\title{
Size effects on free vibration of heterogeneous beams
}

\author{
Bahman Hassanati, ${ }^{1, *}$, Marcus Wheel ${ }^{1}$ \\ ${ }^{1}$ Mechanical \& Aerospace Engineering Department, University of Strathclyde, 75 Montrose Street, Glasgow G1 1XJ, Scotland, UK
}

\begin{abstract}
In this paper the influence of microstructure on the free vibration of geometrically similar heterogeneous beams with free-free boundary conditions was numerically investigated by detailed finite element analysis (FEA) to identify and quantify any effect of beam size on transverse modal frequencies when the microstructural scale is comparable to the overall size. ANSYS Mechanical APDL was used to generate specific unit cells at the microstructural scale comprised of two isotropic materials with different material properties. Unit cell variants containing voids and inclusions were considered. At the macroscopic scale, four beam sizes consisting of one, two, three or four layers of defined unit cells were represented by repeatedly regenerating the unit cell as necessary. In all four beam sizes the aspect ratio was kept constant. Changes to the volume fractions of each material were introduced while keeping the homogenized properties of the beam fixed. The influence of the beam surface morphology on the results was also investigated. The ANSYS results were compared with the analytical results from solution to Timoshenko beam and nonlocal Timoshenko beam as well as numerical results for a Micropolar beam. In nonlocal Timoshenko beams the Eringen's small length scale coefficients were estimated for some of the studied models. Numerical analyses based on Micropolar theory were carried out to study the modal frequencies and a method was suggested to estimate characteristic length in bending and coupling number via transverse vibration which verifies the use of Micropolar elasticity theory in dynamic analysis.
\end{abstract}

\section{Introduction}

In recent years the progress in technologies such as aerospace, biomedical, nanotechnology etc. have demanded the need for the application of small scale structures and that has created a whole new era for researchers to investigate the dynamic behaviour of structures where the classical theories of elasticity become increasingly invalid to use in cases such as small scale heterogeneous beams. Heterogeneity in general is known as a discontinuity of physical properties of material in either a specific direction or multidirectionally. In the literature generally the homogenisation methods are sought to represent the properties of materials with a selected unit cell. Rabboh et al used the rule of mixture to calculate the elastic constants and Poisson's ratio for functionally graded material sandwich beam and investigated the effect of functionally graded material (FGM) on the beam's dynamic performance[1]. Della and Shu used Eshelby's equivalent inclusion method to investigate the vibration of piezoelectric beams and their analytically obtained results also indicate that the size effect due to the size of piezoelectric inclusions, their location in the beam and their volume fraction influence their dynamic behaviour[2].

Homogenisation methods become severely problematic where the size of constituent materials such as inclusions and/or voids becomes comparable to the overall size of the specimen. Modifications to classical elasticity theories are only useful as long as the internal length scale parameters are considered very small. The size dependent characteristics and behaviour of materials have been reported by many researches such as in the work by Groh \& Weaver[3], Gherlone[4], and Schulze et al in laminated beams[5]. Alghamdi and Dasgupta's results in modelling active damping of adaptive structures mostly shows how the beam's time to decay changes by changing the device aspect ratio, inclusion shape, location, and volume fraction, and also how changes in host stiffness results in changes in time to decay and electrical field[6]. Timoshenko beam theory is widely used by researches as it is able to include rotary inertia and shear deformation and therefore it is considered nonlocal if Eringen's small scale effect is incorporated in the governing equations[7]. C M Wang et al used finite segments method to calibrate Eringen's small length scale coefficient for initially stressed vibrating nonlocal beams and stated that ' $\mathrm{e}_{0}$ does not depend on buckling or vibration modes' [8]. In our FE results, however, we observed that in such cases that $\alpha=$ $\left(\mathrm{e}_{0} \mathrm{a}\right)$ is not size independent and therefore it cannot be accepted as a unique property of the material. Available results on the influence of size effect on the behaviour of heterogeneous materials reported by researches show deviation from elastic theories in static loading cases when the beam or plate $\mathrm{L} / \mathrm{h}$ ratio reduces [9]-[15]. Nakamura and Lakes used a two dimensional FE method

\footnotetext{
* Corresponding author: bahman.hassanati@strath.ac.uk
} 
and studied the localised end loads on a strip sample and concluded that as the characteristic length increases, the rate of decay of stress and strain energy reduces. Micropolar theory, however, takes into account couple stresses and thus requires a characteristic length constitutive parameter and an additional degree of freedom, a micro rotation. Micropolar theory by nature incorporates the size effect in the material. A 2D Micropolar strip loaded at one end was investigated by Nakamura \& Lake [16] and the influences of elastic constants especially coupling number and characteristic length are investigated. They concluded that for a very small characteristic length (in comparison with the strip's width), the rate of stress/or strain energy decreases as the characteristic length increases. In the dynamic case this may be shown by wave dispersion. Their work predominantly includes studying the models for various characteristic length and coupling number, $\mathrm{N}$, and provides no method to determine them. However in another work by Lakes, a comprehensive comparison has been made between various theories such as Eringen's nonlocal theory and Micropolar theory[17]. This indicates that the elastic constants can be determined via a dynamic wave propagation method. Nevertheless, in any method such as a size effect based method, field method and/or wave method, there are limitations with regard to the smallest characteristic length. Also, special attention must be given to the coupling number, N, near the limits of zero and one while performing numerical analysis otherwise numerical errors in computation may result.

Wheel et al studied size effects in heterogeneous beams in static cases when loaded in 3 point bending [16]. They investigated size effects in beams with voids and showed that sample stiffness relates to the sample size, as measured by the reciprocal of its depth squared, in a linear manner. They also reported that there are both negative and positive effects of beam size depending on the beam boundary morphology [15]. Waseem et al also investigated the influence of void size on the constitutive properties of a perforated ring model and by relating the diametrical loads, displacement and strain energy derived the final equation relating the stiffness to the specimen size[14]. In the case of smooth specimen surface (rings circumference), the stiffness changes linearly with sample size measure. McGregor provides the same conclusions[18]. Beveridge et al investigated the Micropolar behaviour of perforated beams in 3 point bending and by using a control volume finite element method and iteration to model static 3 point bending test results determined the coupling number for the models[10].

\section{Finite Element Analyses}

\subsection{FE modelling}

Three types of 2D beams were modelled: perforated beams (beams with voids), beams with compliant inclusions and beams with a compliant matrix. Modal analysis was carried out using ANSYS APDL version 16.2. Unit cell variants containing voids and inclusions were considered. The height and length of unit cells are 0.866 and $1 \mathrm{~mm}$. Parametric APDL codes were written to regenerate models for various voids/inclusions volume fractions.
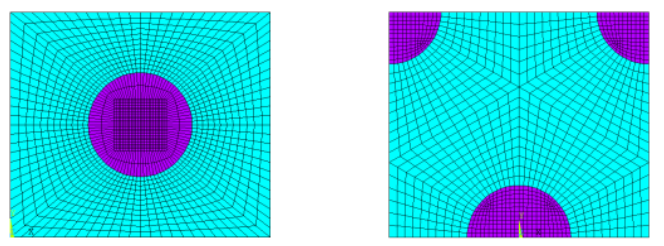

Figure 1: Unit cell consisting of two isotropic materials $r=0.2$ $\mathrm{mm}$, cell on the right is for when inclusions intercept the beams surface.

At the macroscopic scale, unit cells were repeatedly regenerated to produce four beam sizes consisting of one, two, three or four layers of cells while keeping the aspect ratio constant at 10.4:1. Changes to the volume fraction of each material were introduced while keeping the homogenized properties of the beam fixed. This method is meant to account for studying the size effect of free vibration for various volume fractions and beam sizes while keeping the mass of the unit cell and beams overall properties constant. The aim is to study frequency changes for various beam sizes and void/or inclusions volume fractions for lateral vibration modes.

\subsection{Size effect}

In figure 2, the normalised non-dimensional frequencies for the first 10 transverse modes based on the reciprocal measure of 4 beam sizes are shown. The blue lines (dotted lines with squared markers) represent the homogeneous case and the results show that in this case the modal frequency $\lambda$ is size independent. The size effect is greatest for beams which are smaller. It is also evident that the size effect is mode dependent as there is a shift in the nature of size effect, after mode 3 changes in void radius causes a decrease rather than an increase in normalised frequencies. In figure 3 , it is shown that beams with compliant inclusions show similar behaviour.

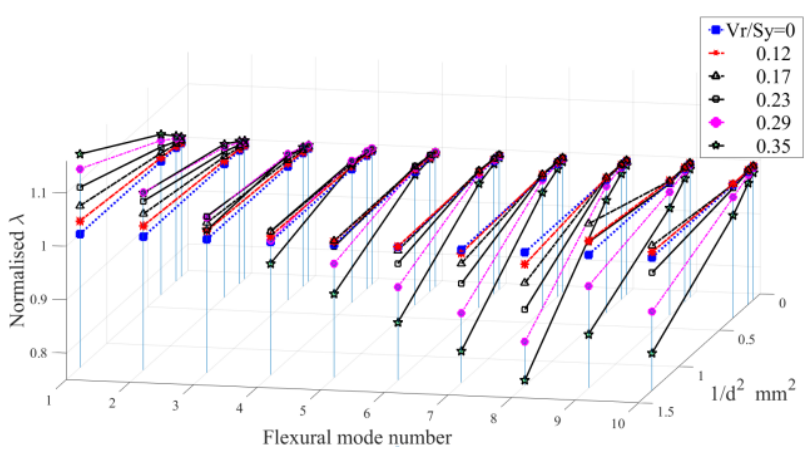

Figure 2: Normalised flexural modal frequencies of the first 10 modes, four beam sizes for beams with voids and smooth morphology 


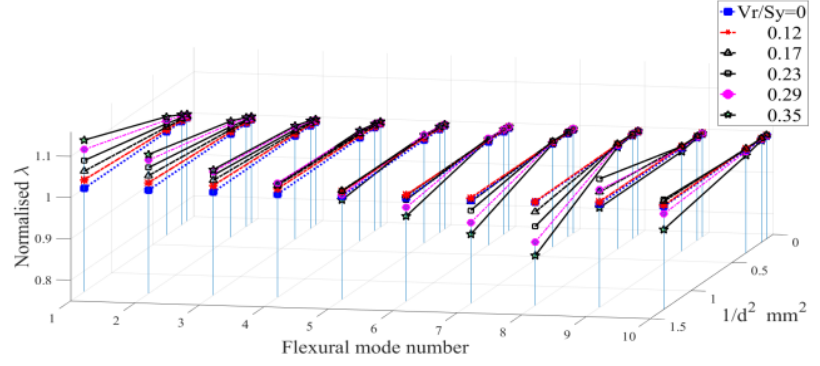

Figure 3: Normalised flexural modal frequencies of the first 10 modes, four beam sizes for smooth surfaced beams with compliant inclusions

From the results we can see that the nature of the size effect changes and $\mathrm{NN}-\lambda$ (Normalised non-dimensional frequency parameter) decreases rather than increases. It has been observed that after some cut-off frequency the FE results increasingly become even more nonlinear and need more research which is beyond the scope of this paper. The results for specimens with stiff inclusions show a different dynamic behaviour, figure 4 , and that it displays a totally different size effect which indicates that increasing volume fraction causes a decrease in NN$\lambda$ and this rate of change decreases at higher mode numbers. However, for beams with voids and compliant inclusions for which the voids and/or inclusions intercept the surfaces, this rate of change increases although the resulting $\mathrm{NN}-\lambda$ remains below the homogenised case.

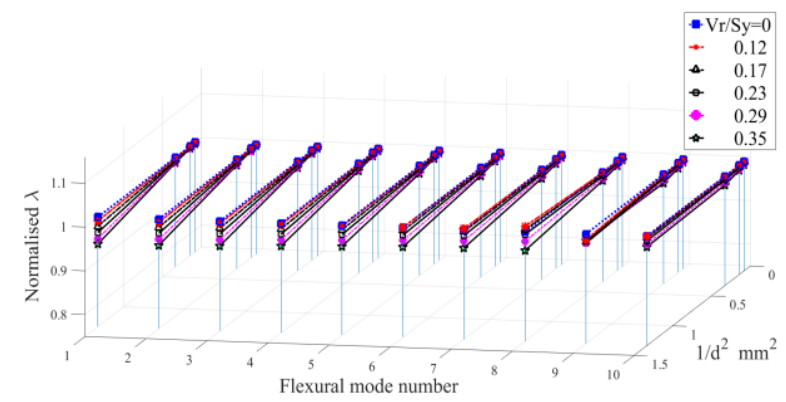

Figure 4: Normalised flexural modal frequencies of the first 10 modes, four beam sizes for smooth surfaced beams with compliant Matrix

\section{Nonlocal Timoshenko Beam}

The nonlocal Timoshenko governing equations can be obtained by applying Hamilton's principle and incorporated the Eringen's small scale coefficient $\alpha=\mathrm{e}_{0} \mathrm{a}$ into the Timoshenko beam model[7]:

$$
\begin{aligned}
& E I \frac{d^{2} \phi}{d x^{2}}-\kappa G A\left(\phi+\frac{d w}{d x}\right)+\rho I \omega^{2} \phi-\left(e_{0} a\right)^{2}\left(\rho A \omega^{2} \frac{d w}{d x}+\right. \\
& \left.\rho I \omega^{2} \frac{d^{2} \phi}{d x^{2}}\right)=0 \\
& \kappa G A\left(\frac{d \phi}{d x}+\frac{d^{2} w}{d x^{2}}\right)+\rho A \omega^{2} w=0
\end{aligned}
$$

where $\phi$ is the rotation, $\mathrm{w}$ is the transverse displacement and $\omega$ is the circular frequency. After decoupling equations (1) and (2) and applying free-free boundary conditions, the following equation may be derived:

$\cosh \beta \cos \gamma+\left(\frac{\left[\left(\mathrm{H}_{2} \mathrm{H}_{3}\right)^{2}-\left(\mathrm{H}_{1} \mathrm{H}_{4}\right)^{2}\right]}{2 \mathrm{H}_{1} \mathrm{H}_{2} \mathrm{H}_{3} \mathrm{H}_{4}}\right) \sinh \beta \sin \gamma=1$

where:

$H_{1}=\alpha^{2} \lambda^{2}+\left(\frac{\alpha^{2} \lambda^{2}}{\xi^{2}}-1\right) \beta \Psi_{\beta}, H_{2}=\alpha^{2} \lambda^{2}+$

$\left(\frac{\alpha^{2} \lambda^{2}}{\xi^{2}}-1\right) \gamma \Psi_{\gamma}$

$H_{3}=\Psi_{\beta}+\beta, H_{4}=\Psi_{\gamma}+\gamma$

$a=\left(1-\frac{\alpha^{2} \lambda^{2}}{\xi^{2}}\right), b=\lambda^{2}\left(\Omega+-\frac{1-\Omega \alpha^{2} \lambda^{2}}{\xi^{2}}+\alpha^{2}\right)$

$c=\lambda^{2}\left(\frac{\lambda^{2} \Omega}{\xi^{2}}-1\right)$

$\left(\begin{array}{l}\beta \\ \gamma\end{array}\right)=\left(\frac{ \pm b+\sqrt{b^{2}-4 a c}}{2 a}\right)^{1 / 2}$

$\Psi_{\beta}=-\frac{\beta^{2}+\lambda^{2} \Omega}{\beta}, \quad \Psi_{\gamma}=\frac{\gamma^{2}+\lambda^{2} \Omega}{\gamma}$

$\bar{x}=\frac{x}{L}, \bar{w}=\frac{w}{L}, \lambda^{2}=\omega^{2} \frac{\rho A L^{4}}{E I}$,

in which $\lambda=\omega^{1 / 2}\left(\rho \mathrm{AL}^{4} / \mathrm{EI}\right)^{1 / 4}$ is the frequency parameter, $\Omega=E I /\left(K_{s} G A L^{2}\right)$ the shear deformation parameter, $\alpha=\mathrm{e}_{0} \mathrm{a} / \mathrm{L}$ a scaling effect parameter and finally $\xi=\mathrm{L}(\mathrm{A} / \mathrm{I})^{1 / 2}$ is the slenderness ratio.

Equation (3) has been solved by the bisection method and the normalised transverse modal frequencies for the first 38 modes of a beam with aspect ratio $\mathrm{L} / \mathrm{d}=10.4$ are provided in figure 5. By comparing these results and the modal frequencies obtained in FE analysis using ANSYS APDL it is possible to obtain Eringen's scale parameter for our heterogeneous models. A direct comparison between the results in figure 5 and the $\mathrm{FE}$ results in section 2.2, confirms that it is not always possible to easily determine $\alpha$ for cases similar to our models. However, by applying a constant $\left(\lambda / \lambda_{0}\right)_{2}=\left(\lambda / \lambda_{0}\right)_{1}-$ $\left[\boldsymbol{C 1} \times\left(\mathbf{1}-\left(\lambda / \lambda_{0}\right)_{\text {Mode_ }}\right)\right]$ and shifting the results below the homogeneous line, it is possible to obtain $\alpha$ for beams with voids and inclusions.

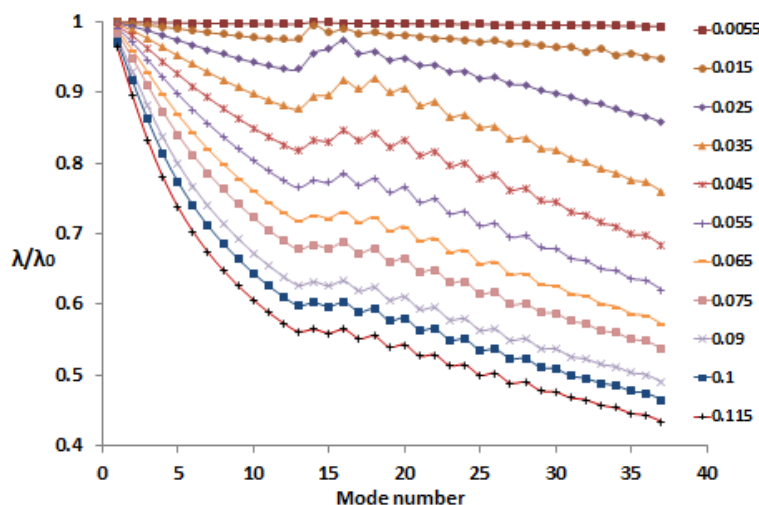

Figure 5: Nonlocal Timoshenko Frequency Parameters for Various $\alpha$ 's, F-F BC 
The values for $\alpha$ via this curve fitting method show ' $\alpha$ ' is not size independent as illustrated in figure 6 therefore it cannot be regarded as a unique property of the material. When voids and inclusions intercept the surfaces, there is no need to consider any constant but the small scale coefficient still showed size dependency.

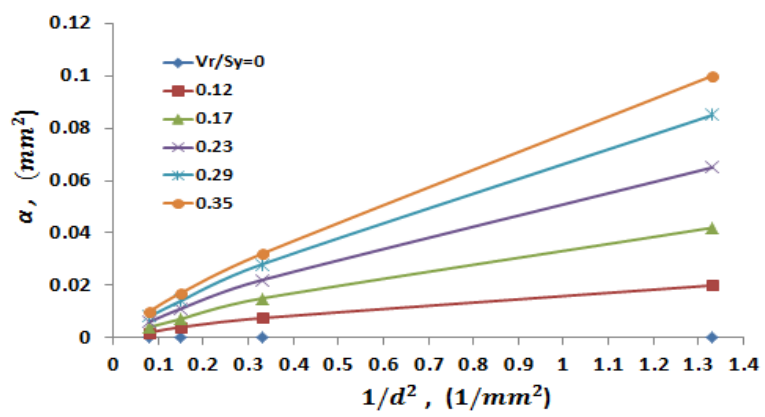

Figure 6: Smooth surfaced perforated beam. Scale Coefficient ' $a$ ' (Alpha), Obtained by curve fitting FE results with NLTB

\section{Micropolar Beam}

\subsection{D Micropolar elastic materials}

The stress-strain relations in classical elasticity may be stated as $\boldsymbol{\tau}_{i j}=\boldsymbol{\lambda} \boldsymbol{\varepsilon}_{\boldsymbol{k} \boldsymbol{k}} \boldsymbol{\delta}_{\boldsymbol{i j}}+\mathbf{2} \boldsymbol{\mu} \boldsymbol{\varepsilon}_{\boldsymbol{i j}}$ where $\lambda$ and $\mu$ are the Lamé constants but in Micropolar elasticity there are four extra elastic constants. In linear, three dimensional, micropolar elasticity the force stresses, $\tau_{\mathrm{ij}}$, and couple stresses, $\mathrm{m}_{\mathrm{ij}}$, are related to the deformations by [17]:

$\tau_{i j}=\lambda \varepsilon_{k k} \delta_{i j}+\left(2 \mu^{*}+\kappa\right) \varepsilon_{i j}+\kappa e_{i j k}\left(\theta_{k}-\emptyset_{k}\right)$

$\boldsymbol{m}_{i j}=\boldsymbol{\alpha} \emptyset_{k}, \boldsymbol{\delta}_{i j}+\boldsymbol{\beta} \emptyset_{i,}+\boldsymbol{\gamma} \emptyset_{j}$

where $\tau$ is the force stress tensor, $\emptyset$ is the micro rotation, $\theta$ is the macro rotation and $\boldsymbol{m}$ is a length scale dependent couple stress. The strain components, $\varepsilon$, are given in terms of the displacements, $u$, and micro rotations, $\emptyset$, by:

$\varepsilon_{i j}=u_{j, i}+e_{j i k} \emptyset_{k}$

Macro rotation and strain tensors are:

$\theta_{k}=\left(e_{i j k} u_{k, \mathrm{j}}\right) / 2, \varepsilon_{i j}=\left(u_{i, j}+u_{j, i}\right) / 2$

where $\mathrm{i}, \mathrm{j}, \mathrm{k}=1,2,3, \delta$ is the Kronecker delta and is equal to 1 if $\mathrm{i}=\mathrm{j}$ otherwise it is zero, $\mathrm{e}_{\mathrm{ijk}}$ the permutation tensor. For even permutation of of $i j k, e_{i j k}=+1$, for odd permutation of $\mathrm{ijk}, \mathrm{e}_{\mathrm{ijk}}=-1$ and otherwise zero.

There are four additional elastic constants $\alpha, \beta, \gamma$ and $\kappa$. However, in 2D Micropolar elasticity the number of constants reduces to 4 independent engineering constants:

$$
\begin{aligned}
E_{m} & =\frac{\left(2 \mu^{*}+\kappa\right)\left(3 \lambda+2 \mu^{*}+\kappa\right)}{\left(2 \lambda+2 \mu^{*}+\kappa\right)}, & v_{m} & =\frac{\lambda}{\left(2 \lambda+2 \mu^{*}+\kappa\right)} \\
l_{b}^{2} & =\frac{\gamma}{2\left(2 \mu^{*}+\kappa\right)} & N^{2} & =\frac{\kappa}{2\left(\mu^{*}+\kappa\right)}
\end{aligned}
$$

$\mathrm{E}_{\mathrm{m}}$ is Micropolar modulus $v_{m}$ is Micropolar Poisson ratio $l_{b}$ is a length scale parameter and it should reflect the microstructural scale. $\mathrm{N}$ is the coupling number and characterises the shear stress asymmetry. In the dynamic case micro inertia also needs to be included. In 2D finite element methods a mass matrix needed to be introduced in to the equations. The asymmetric components of the shear stress:

$G_{11}=\mu^{*}+\kappa, G_{12}=\mu^{*}, \mathrm{G}_{11}=\mathrm{G}_{22}$ and $\mathrm{G}_{12}=\mathrm{G}_{21}$

$G_{s y}=\left(G_{11}+G_{12}\right)=2 \mu^{*}+\kappa, G_{a s y}=G_{11}-G_{12}=\kappa$

are controlled by $\mathrm{N}$ as noted which can therefore be identified at higher order modes involving increased shear deformation using an iteration method that will be described.

\subsection{Micropolar elastic constants}

\subsubsection{Characteristic length in bending, $I_{b}$}

$\mathrm{E}_{\mathrm{fm}}$ and $\boldsymbol{\nu}_{\mathrm{m}}$ can be determined from static tensile tests. $\boldsymbol{l}_{\boldsymbol{b}}$ can be obtained from mode 1 behaviour and equation 1 for $\lambda_{\text {mode }=1}$. In a slender beam where $d / L \ll 1$

Microrotation $\emptyset_{z} \cong$ Macrorotation $\theta_{z}$

Moment, $\mathrm{M}$, is driven by internal force stress, $\tau_{x x}$, and couple stress, $m_{x z}$, Thus:

$M=\int_{A}\left(y \tau_{x x}+m_{x z}\right) d A$

$\frac{1}{R}=\frac{d \theta}{d x}=\frac{d \emptyset_{z}}{d x}=-\frac{d^{2} W}{d x^{2}}$ and: $m_{x z}=\gamma \frac{d \emptyset_{z}}{d x}, \tau_{x x}=\frac{E_{f m} y}{R}$ If $\emptyset_{z} \cong \theta_{z} \rightarrow m_{x z}=\frac{\gamma}{R}, I=\int_{A} y^{2} d A, A=\int_{A} d A$

Substituting for $\frac{1}{R}=-\frac{d^{2} W}{{d x^{2}}^{2}}$ in the moment curvature relationship:

$\frac{d^{2} W}{d x^{2}}=-\frac{M}{E_{m f^{I}+\gamma A}}=-\frac{M}{D_{m f}}$

where $\mathrm{D}_{\mathrm{mf}}=\mathrm{E}_{\mathrm{mf}} \mathrm{I}+\gamma \mathrm{A}=$ Micropolar flexural rigidity (8)

From an unloaded Euler-Bernoulli beam in the dynamic case:

$D_{m f} \frac{d^{4} W}{d x^{4}}-\mu \omega^{2} W=0, \mu=\rho A$

$\omega=\lambda^{2} \sqrt{\frac{D_{m f}}{\rho A L^{4}}} \rightarrow \omega=\lambda^{2} \sqrt{\frac{E_{m f} I+\gamma A}{\rho A L^{4}}}$

$I=\frac{b d^{3}}{12}, A=b d, \gamma=\frac{E_{m f} l_{b}^{2}}{12}$

$\omega^{2}=\frac{E_{f m} \lambda^{4}}{12 \rho L^{4}}\left(d^{2}+l_{b}^{2}\right)$

$D=E I$ In absence of couple stress

$m \cdot \omega^{2}=\frac{E_{f m} \lambda^{4} b}{12}\left(\frac{d}{L}\right)^{3}\left(1+\left(\frac{l_{b}}{d}\right)^{2}\right) \quad \frac{\mathrm{Kg}}{\mathrm{s}^{2}}$ 
Equation (12) can provide valuable information. It relates characteristic length, $\mathrm{l}_{\mathrm{b}}$, non-dimensional frequency, $\lambda$, flexural Micropolar modulus, $\mathrm{E}_{\mathrm{fm}}$, on the right hand side to the beam's mass multiplied by squared frequency on the left hand side. In fact, if the mass times frequency squared can be calculated for beams with various sizes and plotted against reciprocal size measure, $\left(1 / \mathrm{d}^{2}\right)$, then it is possible to obtain the $\mathrm{E}_{\mathrm{fm}}$ or $\lambda$ from the line intercept and the characteristic length from the line slope. As equation (12) is obtained based on the assumptions of slender beam, therefore, the equation is valid for a slender beam and hence mode one only.

\subsubsection{Coupling number, $N$}

If $\alpha, \beta, \gamma$ and $\kappa=0$, then the solid should behave in a classical manner while if $\mathrm{N}=1$ and therefore, microrotation and macrorotation are exactly equal which means they are not kinematically distinct, $\phi_{\mathrm{z}} \simeq \theta_{\mathrm{z}}$.

Having obtained the characteristic length in bending and based on the results from ANSYS FE analysis and iteration process the coupling number, $\mathrm{N}$, may be estimated. The iteration is based on linear regression[10] and fits the curves in the graphs for $m \cdot \omega^{2}$ vs mode numbers (or wavelength). In the iteration process the first three transverse modes were used to iterate for coupling number, $\mathrm{N}$; the reason is to obtain a coupling number which can satisfy all modal frequencies and model depths. It was found that $\mathrm{N}$ changes with void and/or inclusions radius linearly. But this change is not significant for our specimens $0.1 \mathrm{~mm}<\mathrm{V}_{\mathrm{r}}$ or $0.1155<$ $\mathrm{V}_{\mathrm{r}} / \mathrm{S}_{\mathrm{y}}$.

A control volume finite element method (CVFEM) Matlab code has been developed by us which incorporates Micropolar theory and is able to model, mesh and perform both static and modal analysis. A second code is also developed which automatically estimates $\mathrm{N}$.

\section{Results}

Using the CVFEM code and linear regression as described in section 4.2.2, a unique value for $\mathrm{N}$ was identified as $\mathrm{N}=0.0528$ for beams with compliant inclusions and $\mathrm{N}=0.0538$ for beams with voids. Characteristic length does not vary with beam size and only depends on volume fraction. See table 1 below:

Table 1. Characteristic length changes with volume fraction

\begin{tabular}{|c|c|c|}
\hline $\begin{array}{c}\text { Void or } \\
\text { Inclusions radius' } \\
\text { volume fraction }\end{array}$ & $\begin{array}{c}\text { lc for } \\
\text { beams with } \\
\text { Voids, mm }\end{array}$ & $\begin{array}{c}\text { Ic for beams with } \\
\text { compliant } \\
\text { inclusions, mm }\end{array}$ \\
\hline $4 \%$ & 0.2717 & 0.2555 \\
\hline $8 \%$ & 0.4139 & 0.3612 \\
\hline $15 \%$ & 0.5432 & 0.4681 \\
\hline $23 \%$ & 0.6522 & 0.5621 \\
\hline
\end{tabular}

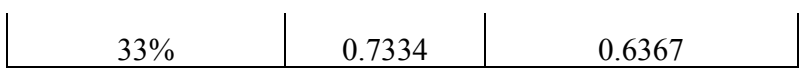

In figures 7 and 8, the finite element results from ANSYS and Micropolar CVFEM codes for mode 1 and 2 are compared after convergence of the iteration process; $m \omega^{2}$ is obtained within two limits of $\mathrm{N}=0$ and $\mathrm{N}=0.9$ ( $\mathrm{N}$ value at higher bound must not equal 1 due to numerical errors it causes.)

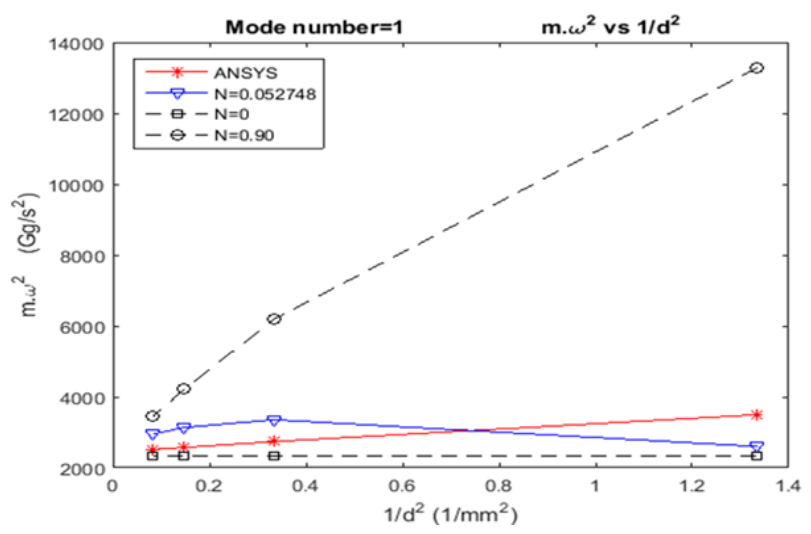

Figure 7: Beams with compliant inclusions, mode 1

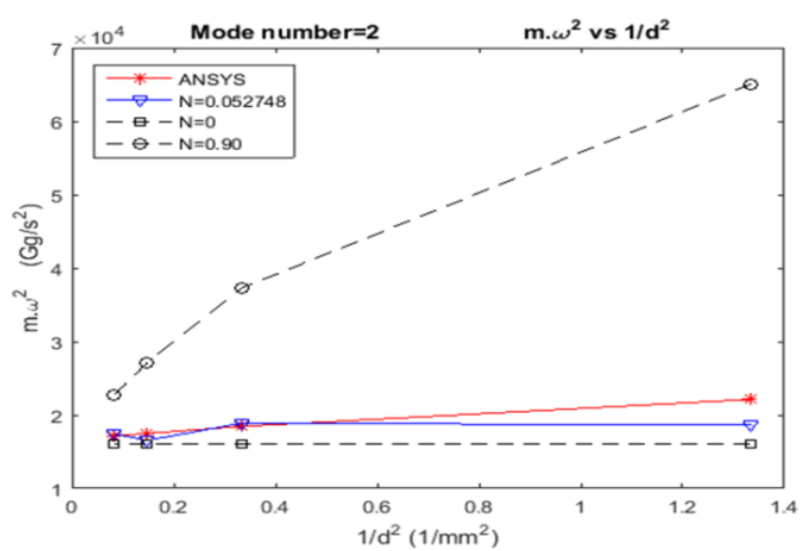

Figure 8: Beams with compliant inclusions, mode 2

In figure 9 the size effect shown in a smooth surfaced beam with voids which is represented by the Micropolar CVFEM code. Beams specifications are:

Model: Beam with voids (Smooth surface), Size: 2, $\mathrm{AR}=10.4, \mathrm{~d}=2 * 0.866 \mathrm{~mm}, \mathrm{DOF}=1107$, ANSYS DOF $=257596, \quad \mathrm{BC}=\mathrm{FF}, \quad \mathrm{N}=0.05, \quad \mathrm{lc}=0.652 \mathrm{~mm}, \quad$ Void radius $=0.25 \mathrm{~mm}$

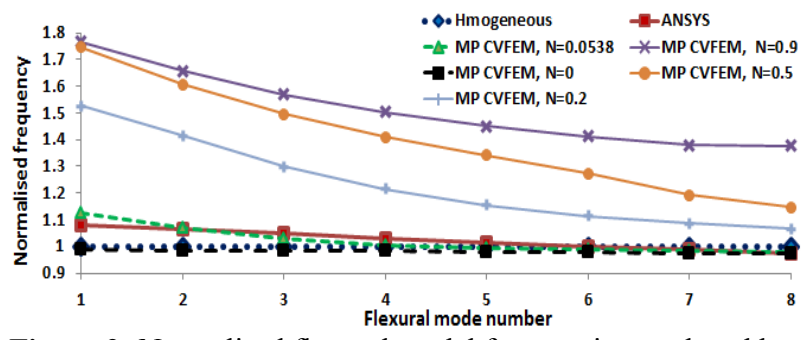

Figure 9: Normalised flexural modal frequencies produced by ANSYS squared markers and CVFEM code shown in dotted line and triangular markers. $l c=0.6522 \mathrm{~mm}, N=0.0538$

Micropolar CVFEM code models homogeneous beams with 6 node triangular elements and characterises the heterogeneity through incorporating the 
characteristic length and coupling number. Comparing the total degrees of freedom, it is clear that CVFEM code uses significantly less DOF's and therefore less computation is needed. Figure 9 also illustrates the sensitivity of frequencies with changes in $\mathrm{N}$ e.g. $\mathrm{N}$ is more sensitive at higher modes, see $\mathrm{N}=0.5$.

\section{Conclusions}

A Micropolar CVFEM code for modal analysis was developed which is able to model, mesh and perform both static and modal analysis using Micropolar theory to account for micro rotation and couple stress. In Micropolar beam vibration, characteristic length in bending is obtained through dynamic analysis. Obtained coupling numbers and Characteristic lengths for models with various void or inclusions volume fraction used in Modal Analysis are fairly accurate and may be used to predict modal frequencies in free vibration with the exceptions of compliant inclusions and/or Voids intersect the beam surface and beams with compliant matrices. The dynamic behaviour of the heterogeneous beams strongly depends to the beam's aspect ratio, mode numbers for which frequencies are obtained, material discontinuity across the height and length of the structure as well as surface morphology. Although Eringen's Nonlocal Timoshenko beam (NTB) could explain the dynamic behaviour of our models to some extent the FE results for our specific models showed that even NTB has shortcomings and is not constant for all model sizes with the same aspect ratio e.g. multiplying the number of constitutive layers require new scaling effect parameter as the layers add up for the models with the same aspect ratios.

\section{References}

1. S. A. Rabboh, N. E. Bondok, T. S. Mahmoud, and H. I. El Kholy, "The Effect of Functionally Graded Materials into the Sandwich Beam Dynamic Performance," vol. 2013, no. November, pp. 751-760, 2013.

2. C. N. Della and D. Shu, "Natural frequency of beams with embedded piezoelectric sensors and actuators," Aerospace, vol. 137, pp. 137-138, 2007.

3. R. M. J. Groh and P. M. Weaver, "On displacement-based and mixed-variational equivalent single layer theories for modelling highly heterogeneous laminated beams," Int. J. Solids Struct., vol. 59, pp. 147-170, 2015.

4. M. Gherlone, "On the Use of Zigzag Functions in Equivalent Single Layer Theories for Laminated Composite and Sandwich Beams: A Comparative Study and Some Observations on External Weak Layers," J. Appl. Mech., vol. 80, no. 6, p.
61004, 2013.

5. S.-H. Schulze, M. Pander, K. Naumenko, and H. Altenbach, "Analysis of laminated glass beams for photovoltaic applications," Int. J. Solids Struct., vol. 49, no. 15-16, pp. 20272036, 2012.

6. A. Dasgupta, "Eigenstrain Techniques for Modeling Adaptive Structures: II. Active Damping," J. Intell. Mater. Syst. Struct., vol. 11, no. 8, pp. 631-641, 2000.

7. C. M. Wang, Y. Y. Zhang, and X. Q. He, "Vibration of nonlocal Timoshenko beams," Nanotechnology, vol. 18, no. 10, p. 105401 , 2007.

8. Z. Zhang, N. Challamel, and C. M. Wang, "Eringen's small length scale coefficient for buckling of nonlocal Timoshenko beam based on microstructured beam model," $J$. Appl. Phys., vol. 114, no. 11, 2013.

9. A. J. Beveridge, M. A. Wheel, and D. H. Nash, "A higher order control volume based finite element method to predict the deformation of heterogeneous materials," Comput. Struct., vol. 129, no. 0, pp. 54-62, 2013.

10. A. J. Beveridge, M. A. Wheel, and D. H. Nash, "The micropolar elastic behaviour of model macroscopically heterogeneous materials," Int. J. Solids Struct., vol. 50, no. 1, pp. 246-255, 2013.

11. A. R. Dehkordi, "3D Finite Element Cosserat Continuum Simulation of Layered Geomaterials," PhD Thesis, p. 263, 2008.

12. J. C. Frame, "A computational and experimental investigation into the micropolar elastic behaviour of cortical bone," PhD Thesis_University Strat., pp. 1199, 2013.

13. A. Riahi and J. H. Curran, "Full 3D finite element Cosserat formulation with application in layered structures," Appl. Math. Model., vol. 33, no. 8, pp. 34503464, 2009.

14. A. Waseem, A. J. Beveridge, M. A. Wheel, and D. H. Nash, "The influence of void size on the micropolar constitutive properties of model heterogeneous materials," Eur. J. Mech. A/Solids, vol. 40, pp. 148-157, 2013.

15. M. a. Wheel, J. C. Frame, and P. E. Riches, "Is smaller always stiffer? On size effects in supposedly generalised continua," Int. J. Solids Struct., vol. 67-68, pp. 84-92, 2015.

16. S. . R. S. L. Nakamura, "Finite element analysis of Saint-Venant end effects in micropolar elastic solids," Adapt. from Eng. Comput. 12, 571-587, pp. 1-10, 1995. 
17. R. Lakes, "Experimental methods for study of Cosserat elastic solids and other generalized elastic continua," Contin. Model. Mater. with Micro-structure, no. 1, pp. 1-22, 1996.

18. M. McGregor and M. A. Wheel, "On the coupling number and characteristic length of micropolar media of differing topology," Proc. R. Soc. A Math. Phys. Eng. Sci., vol. 470, no. 2169, pp. 20140150-20140150, 2014. 